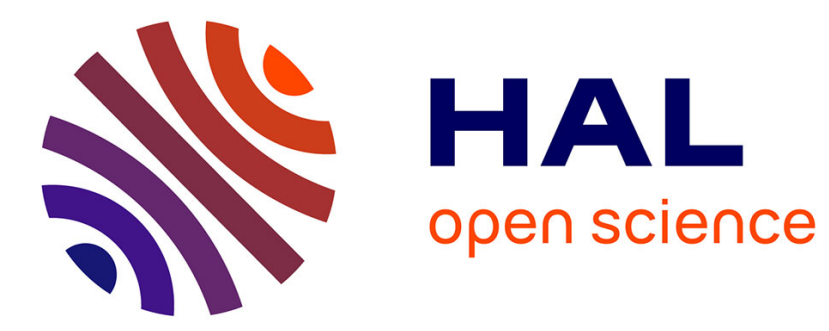

\title{
Flaticon pulses in optical fibers
}

Bastien Varlot, Stefan Wabnitz, Julien Fatome, Guy Millot, Christophe Finot

\section{To cite this version:}

Bastien Varlot, Stefan Wabnitz, Julien Fatome, Guy Millot, Christophe Finot. Flaticon pulses in optical fibers. NonLinear Photonics, Jul 2014, Barcelona, Spain. pp.NTh4A.1. hal-01001734

\section{HAL Id: hal-01001734 https://hal.science/hal-01001734}

Submitted on 2 Aug 2014

HAL is a multi-disciplinary open access archive for the deposit and dissemination of scientific research documents, whether they are published or not. The documents may come from teaching and research institutions in France or abroad, or from public or private research centers.
L'archive ouverte pluridisciplinaire HAL, est destinée au dépôt et à la diffusion de documents scientifiques de niveau recherche, publiés ou non, émanant des établissements d'enseignement et de recherche français ou étrangers, des laboratoires publics ou privés. 


\title{
Flaticon pulses in optical fibers
}

Bastien Varlot, ${ }^{1}$ Stefan Wabnitz, ${ }^{2}$ Julien Fatome, ${ }^{1}$ Guy Millot, ${ }^{1}$ and Christophe Finot ${ }^{1, *}$

${ }^{1}$ Laboratoire Interdisciplinaire Carnot de Bourgogne (ICB), UMR 6303 CNRS/Université de Bourgogne, Dijon, France

${ }^{2}$ Department of Information Engineering, Università di Brescia, Brescia, Italy

*christophe.finot@u-bourgogne.fr

\begin{abstract}
We experimentally investigate the nonlinear reshaping of a continuous wave which leads to chirp-free and flat-top intense pulses or flaticons exhibiting strong temporal oscillations at their edges and a stable self-similar expansion upon propagation of their central region.

OCIS codes: (060.4370) Nonlinear optics, fibers; (190.4380) Nonlinear optics, four-wave mixing;
\end{abstract}

\section{Introduction}

Since the birth of nonlinear optics, there has frequently been a cross-fertilization with hydrodynamics in the study of nonlinear wave propagation phenomena. Examples where this analogy holds are numerous: breakers on a sloping beach and optical wave breaking and shocks, self-filamentation of light beams and wave train disintegration in deep water, dark soliton pulses in optical fibers and now in surface water waves as well, extreme water waves demonstrated and explained in optics. In shallow water, the crossing of currents that propagate with opposite directions may lead to the formation of high-elevation and steep humps of water (or sneaker waves) that could result in severe coastal damages. The analogous effect may also occur in optical fibers: in Refs. [1,2] it was numerically and analytically shown that a continuous-wave (CW) light beam subject to an initial step-wise periodic frequency modulation evolves, upon propagation in a fiber with normal dispersion, towards a train of intense, stable and chirp-free optical pulses. In this case, the physical mechanism leading to pulse formation is the collision among the slower, positively chirped leading wavefront with the faster, negatively chirped trailing wavefront. Given the high degree of flatness of these nonlinear structures, we name them flaticons. Quite remarkably and as predicted in [2], flaticons experience a stable self-similar evolution, undergoing a linear expansion of their temporal width while maintaining their peak-power constant.

In this work we describe the first experimental generation of flaticons in optical fibers, by exploiting the nonlinear and dispersive reshaping of an intense, periodically phase modulated CW beam $[1,3]$.

\section{Experimental setup}

The experimental setup for demonstrating flaticon generation is depicted in Fig. 1, and it relies exclusively on commercially available components ready for telecommunication use. The setup can be divided into two parts. The first part is aimed to imprint on a CW signal the required phase modulation. In order to obtain a modulation amplitude well above the $\pi$ radians value which is routinely provided by phase modulators targeting telecommunication applications, we took advantage of the phenomenon of cross-phase modulation (XPM) imposed by an intensity modulated pump wave upon a CW seed in a 1-km long highly nonlinear optical fiber (HNLF) with low normal dispersion. The pump wave was modulated by an intensity modulator driven by a sinusoidal electrical clock. The pump was also phase modulated at a low frequency in order to avoid any detrimental Brillouin backscattering. A second intensity modulator driven by a deterministic non-return to zero pattern leads to packets of $800 \mathrm{ps}$ duration, repeating every $2.56 \mathrm{~ns}$. This enables to achieve, for a given average power, a more than three-fold increase of the peak power. In order to compensate for the optical losses of the different modulators and to limit the build-up of amplified spontaneous emission, an erbium doped fiber preamplifier was inserted, followed by an optical bandpass filter (OBPF). A high power EDFA (HP EDFA) was then used to obtain the desired power level at the HNLF input, where the pump is combined with the CW seed. At the output of the HNLF, the seed was spectrally isolated by an OBPF. Thanks to XPM, a phase modulation as high as 12 rad can be obtained for an average pump power of $0.6 \mathrm{~W}$.

The second part of the experimental setup relies on a HP EDFA followed by a 10-km long non-zero dispersion shifted fiber (NZ-DSF) with a dispersion $\beta_{2}$ of $5 \mathrm{ps}^{2} / \mathrm{km}$ at $1550 \mathrm{~nm}$, a nonlinear coefficient $\gamma$ of 1.7 $\mathrm{W}^{-1} \cdot \mathrm{km}^{-1}$, and a linear attenuation of $0.2 \mathrm{~dB} / \mathrm{km}$. At the output of the fiber, an optical sampling oscilloscope (OSO) with a picosecond time resolution was used to directly record the temporal intensity profile of the generated train of flaticon pulses.

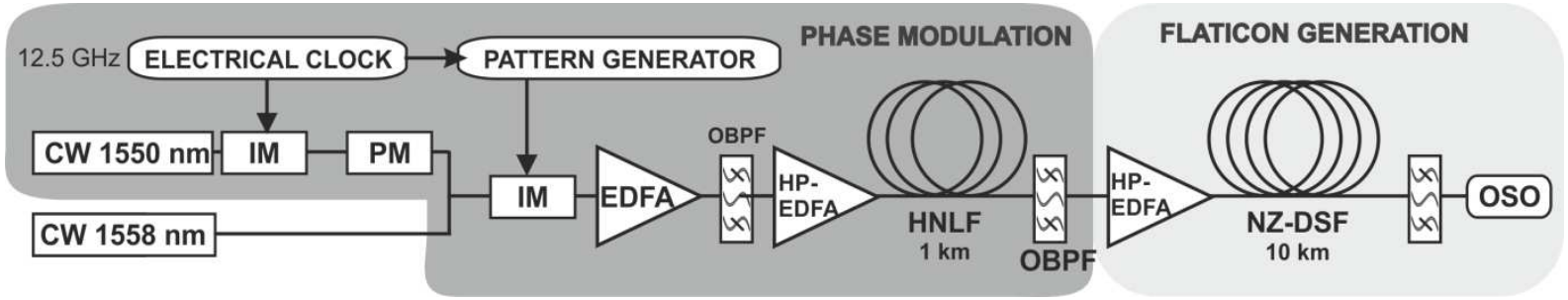

Fig. 1. Experimental setup. 


\section{Experimental results}

Results obtained for three input signal power levels injected into the NZ-DSF are plotted in Fig. 2, and are compared with the corresponding numerical solutions of the NLSE. The experimental results are in pretty good agreement with the numerical predictions, and clearly exhibit several of the important features of optical flaticons. First, we may point out that pulses obtained at a sufficiently high power level clearly exhibit the remarkable flattened temporal intensity profile with high-frequency temporal oscillations in the wings. The resulting nonlinear wave structure sits over a nonzero background: the peak power of the pulse is significantly higher than the background level. The contrast of the experimental oscillations is however reduced when compared with the values expected from numerical simulations. We attribute this contrast reduction to the finite temporal resolution of the OSO, as well as to the influence of residual noise induced by the three cascaded amplifiers in the setup. At low powers, the critical frequency $f_{c}$ defined as $f_{c}=\sqrt{\gamma P / \beta_{2}} / \pi$ is reduced, so that the total frequency jump $f_{s}$ (i.e. two times the product of the repetition rate by the amplitude of the phase modulation $\varphi_{s}$ ) exceeds $f_{c}$. In this context, as predicted in [1], no stable flaticons can be generated.
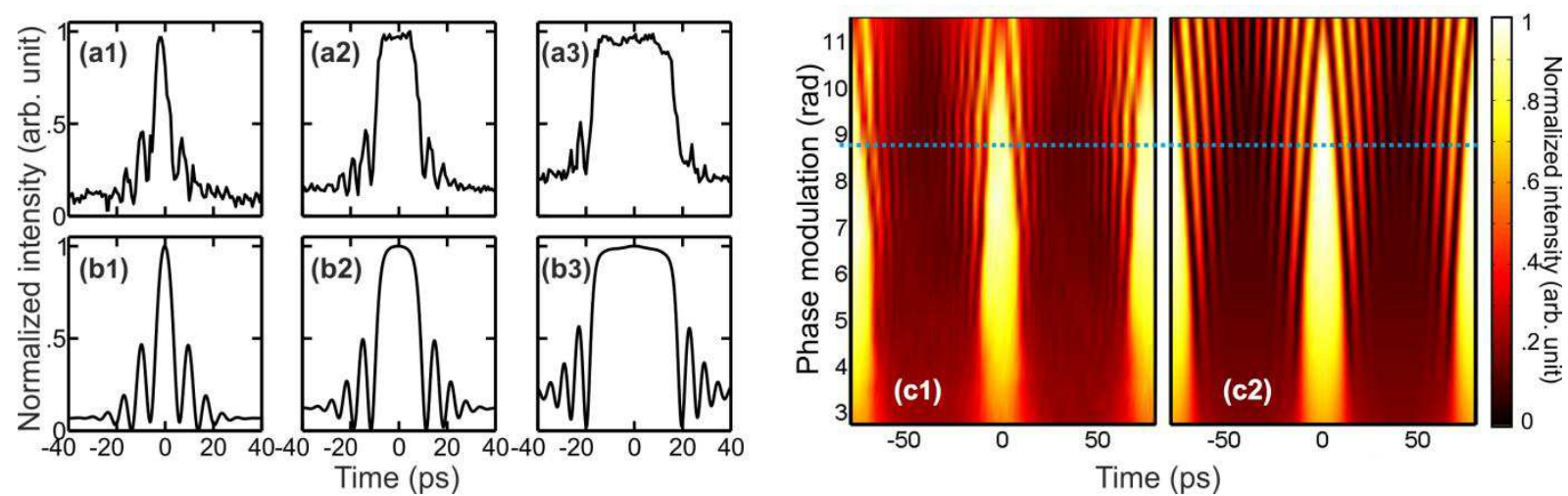

Fig. 2. (a-b) Temporal intensity profiles at the output of the NZ-DSF obtained with a phase modulation of 6.75 rad, and for three different input powers: 1 ) 19 $\mathrm{dBm}, 2) 24 \mathrm{dBm}$ and 3) $28 \mathrm{dBm}$. Experimental results (subplots a) are compared with results of numerical integration of the NLSE (subplots b). (c) Evolution of the temporal intensity profile as a function of the amplitude of the initial phase modulation. Experimental results (panel c1) are compared with numerical simulations (panel c2). The blue dotted line denotes the phase amplitude leading to the frequency jump of $2.4 f_{c}$.

By changing the pump power injected into the HNLF, we have also investigated the influence of the initial level of phase modulation amplitude, for a fixed $250 \mathrm{~mW}$ signal power into the NZ-DSF. Different regimes can be observed, as outlined in Fig. 2(c1). For weak input phase modulations, the typical oscillations expected in the tails of the flaticon are not observed. For a phase modulation amplitude between 5 and 8 rads, clear signatures of flaticon pulses are revealed. For modulation amplitudes above 10 rads, the resulting frequency jump $f_{s}$ becomes too high, leading to an unstable pulse shape. This value can be linked to the threshold of $2.4 f_{c}$ which is estimated to 8.9 rads in the absence of losses [1]. All of these observations are fully consistent with the corresponding numerical simulations summarized on Fig. 2(c2).

\section{Conclusion}

We carried out the first experimental demonstration of the generation of flaticon pulses in a normally dispersive optical fiber. Nonlinear reshaping of an input phase modulated CW leads to the emergence of intense pulses with a flat top lying over a continuous background. The experimental results reveal strong oscillations in the edges of these pulses, as well as a self-similar evolution of the central part of the pulses. Our experiment confirms that an initial step-wise frequency modulation is not a mandatory requirement: flaticon pulses can also emerge from a sinusoidal phase modulation. The reported generation of optical flaticons represents the key building block that may further permit for the observation of optical shallow water rogue waves. Finally we may expect that, similarly to the Peregrine solution, this research paves the way to new investigations in the field of hydrodynamics to help to understand the mechanism for the generation of sneaker waves.

\section{References}

[1] S. Wabnitz, C. Finot, J. Fatome, and G. Millot, "Shallow water rogue wavetrains in nonlinear optical fibers. Phys. Lett. A 377, 932-939 (2013)

[2] G. Biondini, and Y. Kodama, "On the Whitham equations for the defocusing nonlinear Schrodinger equation with step initial data. J. Nonlinear Sci. 16, 435-481 (2006).

[3] B. Varlot, S. Wabnitz, J. Fatome, G. Millot, C. Finot,. "Experimental observation of optical flaticon pulses," Opt. Lett. 38, 3899-3902 (2013). 